\title{
A CLASS OF SPECTRAL SETS
}

\author{
C. ROBERT WARNER
}

ABstract. The two main results are:

(i) If the union and intersection of two closed sets are Ditkin sets, then each of the sets is a Ditkin set.

(ii) If the union of two sets is a spectral set and their intersection is a Ditkin set, then each of the sets is a spectral set.

A corollary of (i) is a generalization of a theorem due to Calderón which proved that closed polyhedral sets in $R^{n}$ are Ditkin (=Calderón) sets. A corollary of (ii) establishes an analogous result for spectral sets.

The proofs hold for commutative semisimple regular Banach algebras which satisfy Ditkin's condition-that the empty set and singletons are Ditkin sets in the maximal ideal space.

Let $G$ be the character group of the locally compact abelian group $\Gamma$, and let $A(G)$ denote the algebra of all Fourier transforms of functions $\varphi$ in $L^{1}(\Gamma)$ with the pointwise product. Thus $f$ belongs to $A(G)$ if and only if $f(s)=\hat{\varphi}(s)$ $=\int_{\Gamma} \varphi(\gamma) \overline{(s, \gamma)} d \gamma$ for some $\varphi$ in $L^{1}(\Gamma)$. If $A(G)$ is equipped with the $L^{1}(\Gamma)$ norm, i.e. $\|f\|=\int_{\Gamma}|\varphi(\gamma)| d \gamma$, then $A(G)$ becomes a commutative Banach algebra isometrically isomorphic to $L^{1}(\Gamma)$.

If $I$ is an ideal in $A(G)$, then $Z(I)$, the zero set of $I$, is the intersection of the zero sets of all the elements $f$ belonging to $I$, i.e. $Z(I)=\bigcap\{Z(f): f \in I\}$. Let $E$ be a closed subset of $G$. The largest ideal (hence closed) in $A(G)$ with zero set $E$ is denoted by $k(E)$, and the smallest ideal with zero set $E$ is denoted by $j(E)$. We note that

$$
k(E)=\{f \in A(G): f=0 \text { on } E\}
$$

and

$$
j(E)=\{f \in A(G): f \text { has compact support disjoint from } E\} .
$$

If $E$ is a closed subset of $G$, then $E$ is a spectral set (a set for which spectral synthesis holds) if the $A(G)$-closure of $j(E)=\overline{j(E)}$ is the only closed ideal with zero set $E[7$, p. 158]. The closed set $E$ is said to be a Ditkin set or a Calderón set [7, p. 169] if, for each $f$ in $k(E), f$ belongs to the closed ideal $\overline{f \cdot j(E)}$. (See also [4, p. 183], [2, p. 227], [5, p. 30], and [3, pp. 513-515].)

The family of spectral sets referred to in the title of this paper is the class of Ditkin sets. It is clear from the definition that every Ditkin set is a spectral set, but it is not known whether or not the two classes coincide. Ditkin sets occur frequently in the study of spectral sets, however. For example, the abstract

Received by the editors July 21, 1975.

AMS (MOS) subject classifications (1970). Primary 43A45, 46J20.

Key words and phrases. Spectral synthesis, Ditkin sets, $C$-sets, Ditkin's condition, regular Banach algebras.

- American Mathematical Society 1976 
Wiener Tauberian theorem follows from the fact that the empty set is a Ditkin set (hence is an $S$-set) and the Silov-Wiener Tauberian theorem can be proved by showing (as in [9]) that closed scattered sets are Ditkin sets. In fact (by [9]), if a set is found to be a spectral set by means of the (generalization of the) Silov-Wiener Tauberian theorem, then it is a Ditkin set.

1. TheOREM. Let $E$ and $F$ be closed subsets of $G$ for which $E \cap F$ is a Ditkin set. Then $E \cup F$ is a Ditkin set if and only if both $E$ and $F$ are Ditkin sets.

Proof. Suppose that $E \cup F$ is a Ditkin set and that $f \in k(E)$.

Since $E \cap F$ is a Ditkin set, there is a sequence $\left\{u_{n}\right\}$ in $j(E \cap F)$ such that $\lim _{n}\left\|u_{n} f-f\right\|=0$. For a fixed $n$, let $C=F \cap$ (support of $u_{n} f$ ). Thus $C$ is compact and disjoint from $E$. Hence there is a $w \in j(E)$ such that $w=1$ on the compact set $C$. Let $g=u_{n} f-u_{n} w f$, so $g$ belongs to $g \cdot j(E \cup F)$. Thus $g$ belongs to $\overline{f \cdot j(E)}$; so $g+u_{n} f w \in \overline{f \cdot j(E)}$. Consequently, $f \in \overline{f \cdot j(E)}$. Therefore $E$ is a Ditkin set. Similarly, $F$ is a Ditkin set.

The other half of the theorem asserts that finite unions of Ditkin sets are Ditkin sets [7, p. 170].

2. Corollary. Let $A$ and $B$ be closed subsets of $G$. If $B$ is a Ditkin set containing $A$, and the relative boundary of $A$ as a subset of $B$ is a Ditkin set in $G$, then $A$ is a Ditkin set.

Proof. Let $E=A$ and $F=\overline{B \backslash A}$ in Theorem 1 .

This result has its origin in Calderón's Theorem [1, Theorem 5]. Calderón proved it for the case where $B$ is a closed subgroup of $G$. The corollary is a generalization of his theorem since closed subgroups are Ditkin sets [ 1 , Theorem 2]. (Also, see [7, p. 170] and [5, pp. 152-153].) The method of Theorem 1 can be employed to prove the following result.

Theorem 1'. If $E \cup F$ is a Ditkin set, and $F \cap$ bdry $E$ is a Ditkin set, then $E$ is a Ditkin set.

3. Lemma. Let $E_{1}$ and $E_{2}$ be closed subsets of $G$ whose intersection is a Ditkin set, and let $E=E_{1} \cup E_{2}$. Let I be a closed ideal in $A(G)$ whose zero set is $E$. Then $I$ can be uniquely expressed in the form $I=I_{1} \cap I_{2}$, where $I_{i}=\overline{I+J_{i}}$, and $J_{i}=\overline{j\left(E_{i}\right)}(i=1,2)$. This form is unique in the sense that if $Z\left(I_{1}^{\prime}\right)=E_{1}, Z\left(I_{2}^{\prime}\right)$ $=E_{2}$, and $I=I_{1}^{\prime} \cap I_{2}^{\prime}$, then $I_{1}^{\prime}=I_{1}$ and $I_{2}^{\prime}=I_{2}$.

Proof. To prove the equality, we have only to show that $I_{1} \cap I_{2} \subseteq I$. Hence, let $f$ belong to $I_{1} \cap I_{2}$ and let $\delta>0$ be arbitrarily chosen. Since $E_{1} \cap E_{2}=F$ is a Ditkin set there is a $v$ in $j(F)$ such that $\|v f-f\|<\delta$.

The function of belongs to $I$ locally at the point at infinity of $G$, and locally at each point of $G$ not in $E \cap(\operatorname{supp} v f)$. Let $C_{1}=E_{1} \cap(\operatorname{supp} v f), C_{2}$ $=E_{2} \cap(\operatorname{supp}$ of $)$, and observe that $C_{1} \cap E_{2}=\varnothing$. Hence there is a $w$ in $j\left(E_{2}\right)$ such that $w=1$ in a neighborhood of $C_{1}$. Since $v f \in I_{1}$, given $\varepsilon>0$, there is a $g$ in $I$ and a $u$ in $j\left(E_{1}\right)$ for which $\|v f w-u w-g w\|<\varepsilon$. The ideal $I$ is closed, and $u w \in I$ so this means that $v f w \in I$. Hence $v f$ belongs to $I$ locally at each point of $C_{1}$, and similarly, it belongs to $I$ locally at each point of $C_{2}$. Consequently of $\in I$. The choice of $\delta>0$ is arbitrary, so it follows that $f \in I$.

To prove the uniqueness, suppose that $Z\left(I_{1}^{\prime}\right)=E_{1}, Z\left(I_{2}^{\prime}\right)=E_{2}$, and that $I_{1} \cap I_{2}=I_{1}^{\prime} \cap I_{2}^{\prime}$. Let $f \in I_{1}^{\prime}$, and $\varepsilon>0$ be given. Since $f \in k(F)$ there is 
a $u$ in $j(F)$ such that $\|u f-f\|<\varepsilon$. Let $K=E_{1} \cap$ (supp $u f$ ), and let $w$ be chosen in $j\left(E_{2}\right)$ such that $w=1$ on a neighborhood of $K$. Thus $u f-u f w \in I_{1}$. But $u f w \in j\left(E_{2}\right) \subset I_{2}^{\prime}$, so $u f w \in I_{1} \cap I_{2}$, i.e. $u f w \in I_{1}$. Since $u f-u f w$ belongs to $I_{1}$ also, it follows that $u f \in I_{1}$, hence that $f \in I_{1}$. Thus $I_{1}^{\prime} \subseteq I_{1}$. Similarly, $I_{1} \subseteq I_{1}^{\prime}$, so that $I_{1}^{\prime}=I_{1}$. By an identical argument it follows that $I_{2}^{\prime}=I_{2}$.

4. THEOREM. Let $E_{1}$ and $E_{2}$ be closed subsets of $G$ whose intersection is a Ditkin set, and let $E=E_{1} \cup E_{2}$. Then $E$ is a spectral set if and only if both $E_{1}$ and $E_{2}$ are spectral sets.

Proof. If $E_{1}$ and $E_{2}$ are $S$-sets and $I=\overline{j(E)}$, then by Lemma 3,

$$
\begin{aligned}
\overline{j(E)} & =I=\overline{I+J_{1}} \cap \overline{I+J_{2}}=\overline{I+k\left(E_{1}\right)} \cap \overline{I+k\left(E_{2}\right)} \\
& =k\left(E_{1}\right) \cap k\left(E_{2}\right)=k(E),
\end{aligned}
$$

that is, $E$ is an $S$-set. Conversely, if $E$ is an $S$-set and $I=\overline{j(E)}$, then $\overline{j(E)}=I=\overline{I+J_{1}} \cap \overline{I+J_{2}}=J_{1} \cap J_{2}$ and $k(E)=k\left(E_{1}\right) \cap k\left(E_{2}\right)=\overline{j(E)}$ by assumption. Hence, by Lemma $3, J_{1}=k\left(E_{1}\right)$, and $J_{2}=k\left(E_{2}\right)$.

5. Corollary. Let $F$ be a spectral set and let $E$ be a closed subset of $F$. If the relative boundary of $E$ as a subset of $F$ is a Ditkin set, then $E$ is a spectral set.

6. Remark. The first half of Theorem 4-that the union of two $S$-sets which intersect in a Ditkin set is an $S$-set-was obtained for $A(G)$ by Herz [2, p. 228], and also, essentially, by Calderón [1, p. 3]. For the more general Banach algebra case, Reiter [6, p. 557] proved Lemma 3 and Theorem 4 in the case where the two sets were disjoint.

Our result, as well as that of [9], was suggested by a study of Reiter's paper [6]. Saeki [8, p. 551] gives an elegant proof in a Banach-algebraic setting of a result which is more general than the first half of Theorem 4. It is not difficult to see that our proofs of Lemma 3 and Theorem 4 can be modified slightly to prove the more general results, Lemma $3^{\prime}$ and Theorem 4'.

LEMmA 3'. Let $E_{1}$ and $E_{2}$ be closed subsets of $G$, let $E=E_{1} \cup E_{2}$, and suppose ihat there is a Ditkin set $C \subset E$ such that the intersection of the boundaries of $E_{1}, E_{2}$ and of $E$ is contained in $C$. Let $I$ be a closed ideal in $A(G)$ with $Z(I)=E$. Then $I$ can be uniquely expressed in the form $I=I_{1} \cap I_{2}$, where $I_{i}=\overline{I+J_{i}}$, and $J_{i}=\overline{j\left(E_{i}\right)}(i=1,2)$. This form is unique in the sense that if $Z\left(I_{1}^{\prime}\right)=E_{1}, Z\left(I_{2}^{\prime}\right)=E_{2}$, and $I=I_{1}^{\prime} \cap I_{2}^{\prime}$, then $I_{1}^{\prime}=I_{1}$ and $I_{2}^{\prime}=I_{2}$.

THEOREM 4'. Let $E_{1}$ and $E_{2}$ be closed subsets of $G$, let $E=E_{1} \cup E_{2}$, and suppose there is a Ditkin set $C \subset E$ such that the intersection of the boundaries of $E_{1}, E_{2}$ and of $E$ is contained in $C$. Then $E$ is a spectral set if and only if both $E_{1}$ and $E_{2}$ are spectral sets.

ACKNOWLEDGEMENT. I would like to acknowledge the hospitality extended to me by the University of California at Irvine while I was engaged in this research.

\section{REFERENCES}

1. A. P. Calderón, Ideals in group algebras, Sympos. on Harmonic Analysis and Related Integral Transforms, Cornell University, 1956 (mimeographed). 
2. C. S. Herz, The spectral theory of bounded functions, Trans. Amer. Math. Soc. 94 (1960), 181-232. MR 24 \#A1627.

3. E. Hewitt and K. A. Ross, Abstract harmonic analysis. Vol. II: Structure and analysis for compact groups. Analysis on locally compact Abelian groups, Die Grundlehren der math. Wissenschaften, Band 152, Springer-Verlag, New York and Berlin, 1970. MR 41 \# 7378; erratum, 42, p. 1825 .

4. J.-P. Kahane and R. Salem, Ensembles parfaits et séries trigonométriques, Actualités Sci. Indust., no. 1301, Hermann, Paris, 1963. MR 28 \#279.

5. H. Reiter, Classical harmonic analysis and locally compact groups, Oxford Univ. Press, Oxford, 1968. MR 46 \#5933.

6. Contributions to harmonic analysis. VI, Ann. of Math. (2) 77 (1963),522-562. MR 27 \#1778.

7. W. Rudin, Fourier analysis on groups, Interscience Tracts in Pure and Appl. Math., no. 12, Interscience, New York, 1962. MR 27 \#2808.

8. S. Saeki, Spectral synthesis for the Kronecker sets, J. Math. Soc. Japan 21 (1969), 549-563. MR 40 \#7733.

9. C. R. Warner, A generalization of the Silov-Wiener Tauberian theorem, J. Functional Analysis 4 (1969), 329-331. MR 40 \#649.

Department of Mathematics, University of Maryland, College Park, Maryland 20742 